\title{
The globular cluster systems of NGC 3258 and NGC 3268 in the Antlia cluster ${ }^{\star}$
}

\author{
B. Dirsch ${ }^{1}$, T. Richtler ${ }^{1}$, and L. P. Bassino ${ }^{2}$ \\ ${ }^{1}$ Universidad de Concepción, Departamento de Física, Casilla 160-C, Concepción, Chile \\ 2 Facultad de Ciencias Astronómicas y Geofísicas, Universidad Nacional de La Plata, Paseo del Bosque S/N, 1900-La Plata, \\ Argentina and CONICET
}

Received 16 April 2003 / Accepted 30 June 2003

\begin{abstract}
The Antlia galaxy cluster is the third nearest galaxy cluster after Virgo and Fornax. We used the wide-field MOSAIC camera of the 4-m CTIO telescope to search in the brightest cluster galaxies for globular cluster systems, which were detected in the two larger ellipticals - NGC 3258 and NGC 3268. These galaxies each contain several thousand clusters; NGC 3258 more than NGC 3268. The color distributions of the globular cluster systems are clearly bimodal. The peak colors agree with those of other ellipticals. The radial number density profiles of the globular cluster systems are indistinguishable for the two galaxies and no difference in the distribution of red and blue clusters - as observed in other elliptical galaxies - can be seen. The light profile of NGC 3268 appears to be similar to that of NGC 1399, the central galaxy of the Fornax cluster. NGC 3258 has a light profile which is steeper at large radii. Both galaxies exhibit color gradients, becoming bluer outwards. In NGC 3268, the color and morphology in the inner $3^{\prime \prime}$ indicate the presence of an inner dusty disk. The globular cluster systems closely trace the galaxy light in the studied radial regime. The elongation of the cluster systems of both galaxies is approximately aligned at large radii with the connecting axis of the two galaxies. We find specific frequencies within a radial range of $4^{\prime}$ of $S_{N}=3.0 \pm 2.0$ for NGC 3268 and $S_{N}=6.0 \pm 2.5$ for NGC 3258 .

As a byproduct resulting from surveying our wide-field frames, we describe a strange absorption feature in the Antlia spiral galaxy NGC 3269, which we argue might be a tiny galactic dust cloud projected onto NGC 3269.
\end{abstract}

Key words. galaxies: elliptical and lenticular, cD - galaxies: individual: NGC 3258, NGC 3268, NGC 3269 galaxies: star clusters - galaxies: stellar content - galaxies: structure

\section{Introduction}

\subsection{The Antlia cluster}

The Antlia galaxy cluster, located in the Southern sky at low Galactic latitude $\left(19^{\circ}\right)$, is after Virgo and Fornax the nearest galaxy cluster. It is described as "a beautiful, small, nearby cluster of Bautz-Morgan type III" (Hopp \& Materne 1985; Nakazawa et al. 2000). As already noted by Hopp \& Materne (1985), there exists a slight confusion in the nomenclature: the Antlia cluster as defined by Sandage (1975) should not be mistaken for the Antlia group defined by Tully et al. (1982) that belongs to the Local Super Cluster.

The galaxy content of the Antlia cluster has been studied by Hopp \& Materne (1985) and by Ferguson \& Sandage (1990, 1991). Ferguson \& Sandage (1990) identified in total 234 Antlia cluster galaxies, compared to 340 in Fornax. After correction for the completeness of the sample and taking the different distances into account, they found that the

Send offprint requests to: B. Dirsch, e-mail: bdirsch@cepheid.cfm.udec.cl

* Based on observations collected at the Cerro Tololo InterAmerican Observatory (CTIO). total number of Antlia cluster galaxies is larger than that of the Fornax cluster within a radial distance of 5 times the core radius (420 versus 330 galaxies).

Of the seven nearby groups/clusters studied by Ferguson \& Sandage $(1990,1991)$, the Antlia cluster has the highest central galaxy number density (e.g. roughly three times larger than in Fornax or Virgo, depending on the assumed distance). In accordance with the large density, its galaxy population is dominated by early-type galaxies. The dwarf ellipticals are by far the most abundant galaxy type in this cluster.

The Antlia cluster's galaxy velocity dispersion ranges from $360 \mathrm{~km} \mathrm{~s}^{-1}$ to $560 \mathrm{~km} \mathrm{~s}^{-1}$, depending on the galaxy sample. One complication is that there might be a connection from the Antlia cluster to the Hydra I cluster that has a higher redshift (3400 $\mathrm{km} \mathrm{s}^{-1}$ versus $2900 \mathrm{~km} \mathrm{~s}^{-1}$ ) (Hopp \& Materne 1985). In addition, it has been found that the early-type galaxies have a systematically lower radial velocity with respect to late-type galaxies, causing some confusion in the velocity dispersion determination (Hopp \& Materne 1985).

The central part of the Antlia cluster consists of two subgroups (see Fig. 1), each dominated by a giant elliptical galaxy. The Northern subgroup contains the early-type galaxy 
NGC 3268 and three larger late-type galaxies. The dominating elliptical of the Southern subgroup is NGC 3258 that is accompanied by two smaller ellipticals. The two dominating ellipticals, NGC 3258 and NGC 3268, nearly have the same luminosity. The Antlia cluster is therefore of a different type than for example the Virgo or Fornax clusters which are dominated by large central galaxies.

The X-ray properties of the Antlia cluster are peculiar. Pedersen et al. (1997) used ASCA observations centered on NGC 3258, where they clearly found extended X-ray emission. They also noted that the $1.7 \mathrm{keV}$ hot gas extends towards NGC 3268 and they excluded this part from their investigation. The emission peak is offset by $1.1^{\prime}$ from NGC 3258 and does not coincide with any other galaxy. They derived a total mass for the NGC 3258 group of $0.9-2.4 \times 10^{13} M_{\odot}$ within $240 \mathrm{kpc}$. The NGC 3268 group has been studied by Nakazawa et al. (2000) using ASCA and ROSAT data both centered on NGC 3268. They confirmed the X-ray emission extending towards NGC 3258 and excluded this direction as well. According to their study Antlia is embedded in a quasi isothermal X-ray halo with a temperature of $2 \mathrm{keV}$. As in Pedersen et al. (1997), no excess central brightness connected with the central galaxy, as frequently seen in nearby clusters, can be detected. This distinguishes the Antlia cluster from clusters like Fornax (Ikebe et al. 1996) or Hydra-A (Ikebe et al. 1997). Nakazawa et al. (2000) used their X-ray observations to derive a total gravitating mass of $1.9 \times 10^{13} M_{\odot}$ within a radius of $250 \mathrm{kpc}$. Strikingly, both groups obtained cluster masses that are of the same order of magnitude (these masses are measured within a radius that encompasses the two central galaxies) as the mass derived for the optically larger Fornax cluster within the same radius (Jones et al. 1997).

While the early-type to late-type galaxy ratio indicates an evolved system, the existence of two subgroups, which also may be present in the overall mass distribution, means that the total system has not yet completed its evolution. We might be witnessing the merging of two small, rather evolved, compact clusters.

Although Antlia is nearby and exhibits interesting properties, the globular cluster systems (GCSs) of the dominating galaxies have not yet been studied. A GCS provides insight into the stellar populations of the host galaxies, where the galaxy light itself has become too faint to be studied in detail, offering single stellar populations. GCSs have also been used to trace the galaxies' evolution (e.g. Pritchet \& Harris 1990; Ashman \& Zepf 1992; McLaughlin et al. 1993; Forbes et al. 1997; Harris et al. 2000; Harris 2001; Beasley et al. 2002; Côté et al. 2002; Beasley et al. 2003). Here we investigate the GCSs of the two dominating ellipticals and study their basic properties, like color and radial number distribution. This is then compared to the light profiles of the host galaxies and their color properties.

The wide-field MOSAIC camera mounted in the prime focus of the CTIO 4-m telescope is the ideal instrument for this task: the main body of the Antlia cluster fits perfectly into one single MOSAIC frame. We used the Washington filter system, since it has a very good metallicity resolution and it is rather powerful in separating compact, blue background galaxies from cluster candidates (Dirsch et al. 2003).

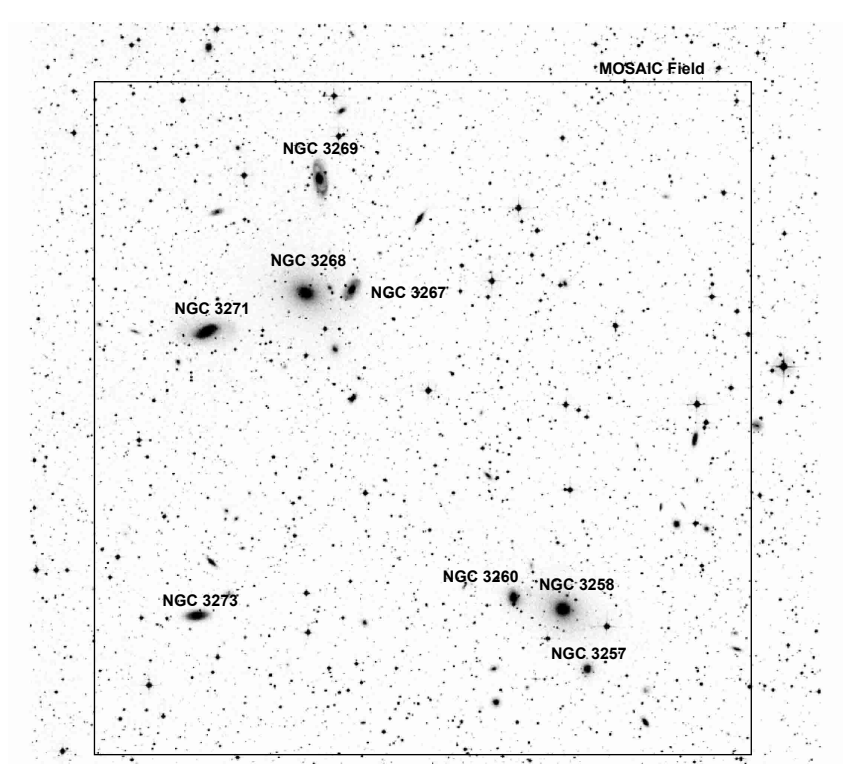

Fig. 1. The MOSAIC field is overlaid on a DSS image of the central part of the Antlia cluster. North is up, East to the left.

\subsection{Distances of NGC 3258 and NGC 3268}

The distances towards NGC 3258 and NGC 3268 have been derived with SBF techniques (Tonry et al. 2001) and are $(m-M)=32.58 \pm 0.27$ and $(m-M)=32.71 \pm 0.25$, respectively. This agrees with the Hubble flow distance if a Hubble constant of $75\left(\mathrm{~km} \mathrm{~s}^{-1}\right) / \mathrm{Mpc}$ is assumed. Prugniel et al. (1996) obtained a distance of $(m-M)=32.92$ for both galaxies using the fundamental plane. However, the extinctions used by Prugniel et al. are taken from Burstein \& Heiles (1982) which are lower by 0.08 and 0.14 in $V$ for NGC 3258 and NGC 3268, respectively, than those of Schlegel et al. (1998), which were used by Tonry et al. Since we prefer the reddening values of Schlegel et al., the distance modulus of Prugniel et al. would be $(m-M)=32.86$ for NGC 3258 and $(m-M)=32.78$ for NGC 3268. The distances towards the two ellipticals are hence the same within the uncertainties and we adopt in the following the mean of the four distance moduli of $(m-M)=$ $32.73 \pm 0.25$ towards the Antlia cluster, so that angular distances of $1^{\prime \prime}$ and $1^{\prime}$ correspond to $170 \mathrm{pc}$ and $10.2 \mathrm{kpc}$, respectively. However, assuming one distance for all Antlia cluster galaxies might be an oversimplification due to the fact that NGC 3271, NGC 3269 and NGC 3267 have very deviating radial velocities (see Table 1) with respect to the other galaxies. Interestingly, these three galaxies are located at very small projected radial distances from NGC 3268, for which the SBF technique gave a larger distance.

\section{Observations and reduction}

The data set consists of Washington wide-field images taken with the MOSAIC camera mounted at the prime focus of the CTIO 4-m Blanco telescope during 4/5 April 2002. We obtained four $600 \mathrm{~s}$ images in $R$ and seven $900 \mathrm{~s}$ images in $C$. In addition, a $60 \mathrm{~s}$ exposure was taken in $R$ and a $180 \mathrm{~s}$ exposure in $C$. The galaxies' cores are not saturated in these short 
Table 1. Basic data about the largest galaxies in the field. The velocities are taken from the RC3 catalogue. The reddening values are taken from Schlegel et al. (1998).

\begin{tabular}{ccccc}
\hline \hline Object & RA $(\mathrm{J} 2000)$ & Dec $(\mathrm{J} 2000)$ & vel. $\left[\mathrm{km} \mathrm{s}^{-1}\right]$ & $E(B-V)$ \\
\hline NGC 3268 & $10^{\mathrm{h}} 30^{\mathrm{m}} 01^{\mathrm{s}}$ & $-35^{\circ} 19^{\prime} 30^{\prime \prime}$ & $2805 \pm 22$ & 0.11 \\
NGC 3258 & $10^{\mathrm{h}} 28^{\mathrm{m}} 54^{\mathrm{s}}$ & $-35^{\circ} 36^{\prime} 22^{\prime \prime}$ & $2792 \pm 28$ & 0.08 \\
NGC 3257 & $10^{\mathrm{h}} 28^{\mathrm{m}} 47^{\mathrm{s}}$ & $-35^{\circ} 39^{\prime} 28^{\prime \prime}$ & $3172 \pm 32$ & 0.08 \\
NGC 3260 & $10^{\mathrm{h}} 29^{\mathrm{m}} 06^{\mathrm{s}}$ & $-35^{\circ} 35^{\prime} 41^{\prime \prime}$ & $2416 \pm 32$ & 0.09 \\
NGC 3273 & $10^{\mathrm{h}} 30^{\mathrm{m}} 30^{\mathrm{s}}$ & $-35^{\circ} 36^{\prime} 37^{\prime \prime}$ & $2429 \pm 66$ & 0.10 \\
NGC 3271 & $10^{\mathrm{h}} 30^{\mathrm{m}} 27^{\mathrm{s}}$ & $-35^{\circ} 21^{\prime} 30^{\prime \prime}$ & $3794 \pm 66$ & 0.11 \\
NGC 3267 & $10^{\mathrm{h}} 29^{\mathrm{m}} 49^{\mathrm{s}}$ & $-35^{\circ} 19^{\prime} 23^{\prime \prime}$ & $3745 \pm 41$ & 0.10 \\
NGC 3269 & $10^{\mathrm{h}} 29^{\mathrm{m}} 58^{\mathrm{s}}$ & $-35^{\circ} 13^{\prime} 24^{\prime \prime}$ & $3799 \pm 41$ & 0.10 \\
\hline
\end{tabular}

exposures. The location of the MOSAIC field, overlaid on a DSS image, is shown in Fig. 1 with the brightest galaxies labelled.

We used the Kron-Cousins $R$ and Washington $C$ filters, although the genuine Washington system uses $T 1$ instead of $R$. However, Geisler (1996) has shown that the Kron-Cousins $R$ filter is more efficient than $T 1$ and that $R$ and $T 1$ magnitudes are closely related, with only a very small color term and zero-point difference (we got $R-T 1=$ -0.02 from standard stars). The MOSAIC wide-field camera images a field of $36^{\prime} \times 36^{\prime}$. The 8 SITe CCDs have pixel scales of $0.27^{\prime \prime} /$ pixel. Further information on the MOSAIC camera can be found in the MOSAIC homepage (http: //www . noao.edu/kpno/mosaic/mosaic.html).

We dithered the data to fill the gaps between the eight individual CCD chips. Due to the dithering the entire area could not be covered by the same number of exposures which restricts the usable field. For the final frames we thus discarded the outer regions that showed a much higher noise than the inner area and trimmed the images to $34.7 \times 34^{\prime} .7$. The seeing on the final $R$ image is $1^{\prime \prime}$ and on the final $C$ image $1^{\prime \prime} 1$.

The MOSAIC data has been handled using the mscred package within IRAF. In particular, this package can correct for the variable pixel scale across the CCD which would cause otherwise a $4 \%$ variability of the brightness of stellar-like objects from the center to the corners. The flatfielding residuals led to sensitivity variations $\leq 2.5 \%$ (peak-to-peak).

In order to facilitate the search for point sources, the extended galaxy light was subtracted. This was done using a median filter with an inner radius of $9.5^{\prime \prime}$ and an outer radius of $11^{\prime \prime}$. This size is large enough for not altering the point source photometry which has been verified with artificial star tests described later on.

The photometry has been done using the PSF fitting routine allstar within DAOPhot II.

For the point source selection we used the $\chi$ and sharpness values from the PSF fit and found approximately 11000 point sources. The brightest non-saturated objects have $T 1 \approx 18$ (depending slightly on the individual MOSAIC chip).

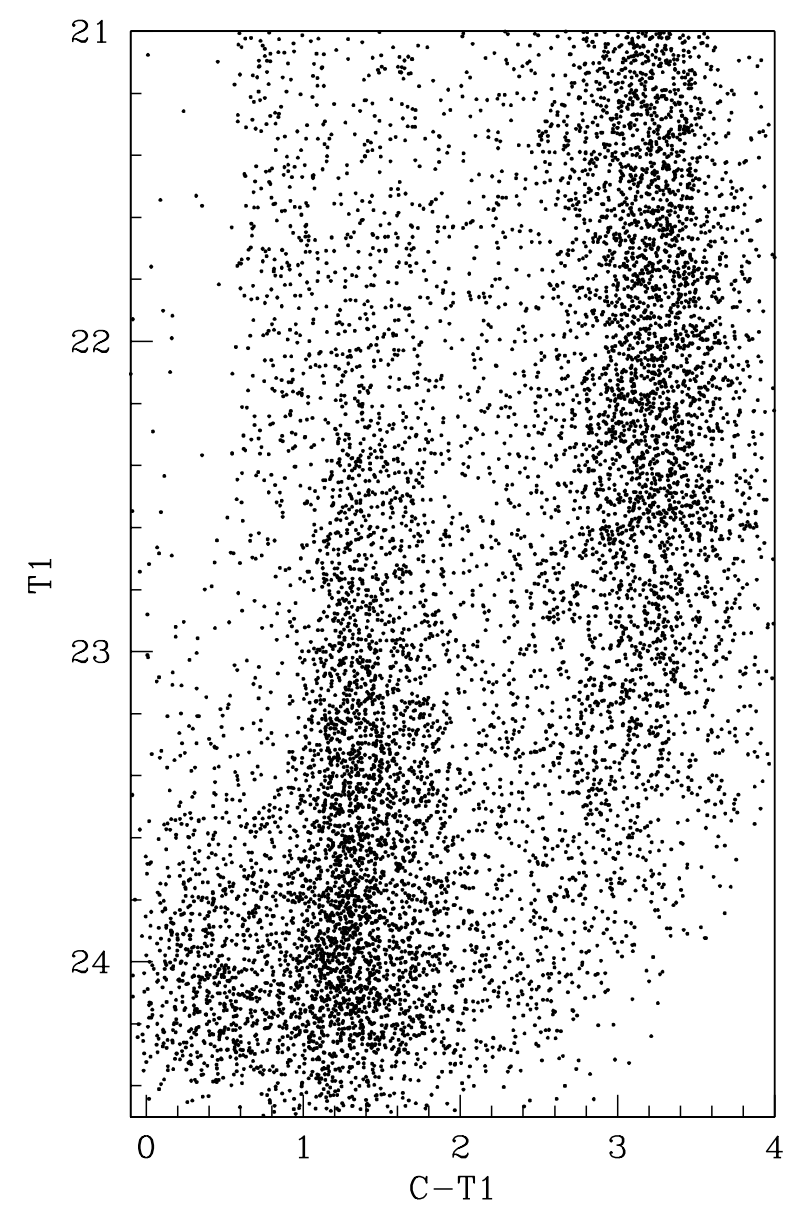

Fig. 2. The color magnitude diagram of all point sources in the MOSAIC field. Globular cluster candidates are known to occupy the color range of approximately $0.9<C-T 1<2.3$. Objects with a color redder than $C-T 1>3$ are in their majority Galactic foreground stars. Objects fainter than approximately $T 1=23.5$ and bluer than $C-T 1=1$ predominately are background galaxies.

\subsection{Photometric calibration}

In each of the 2 nights 4 to 5 fields, each containing about 10 standard stars from the list of Geisler (1996a), have been observed with a large coverage of airmasses (typically from 1.0 to 1.9). It was possible to use a single transformation, since the coefficients were indistinguishable within the uncertainties during these nights.

We derived the following relations between instrumental and standard magnitudes:

$$
\begin{aligned}
T 1= & +(0.71 \pm 0.01)-(0.07 \pm 0.01) X_{R} \\
& +(0.033 \pm 0.003)(C-T 1) \\
(\mathrm{C}-\mathrm{T} 1)= & (c-r)-(0.74 \pm 0.02)-(0.20 \pm 0.01) X_{C} \\
& +(0.088 \pm 0.004)(C-T 1)
\end{aligned}
$$

The standard deviation of the difference between instrumental and calibrated magnitudes is 0.021 in $T 1$ and 0.023 in $C-T 1$.

The color magnitude diagram for all point sources in the MOSAIC images is plotted in Fig. 2. 

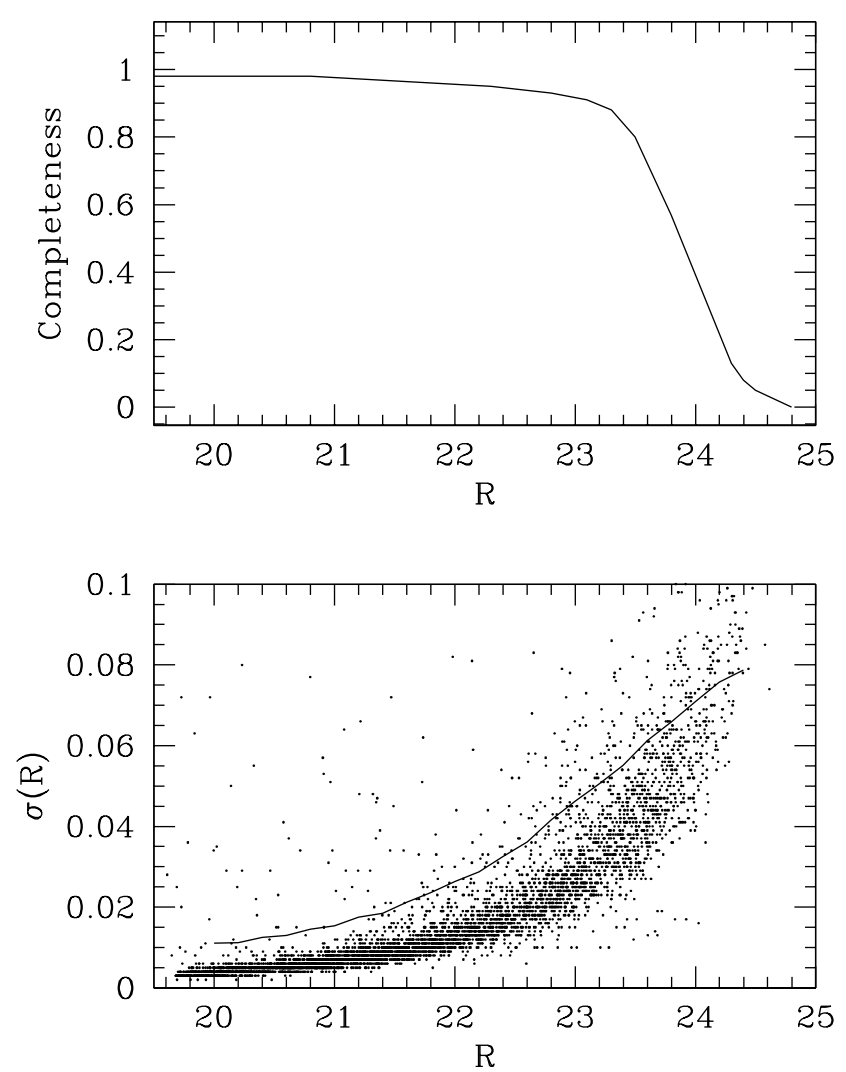

Fig. 3. In the upper panel the completeness function for globular cluster candidates $(0.9<(C-T 1)<2.3)$ is shown. In the lower panel the DAOPhot errors (dots) are compared to the mean standard deviation between the input and output magnitude of the artificial stars (solid line).

The reddening towards the Antlia cluster varies according to Schlegel et al. (1998) between $E_{B-V}=0.08$ and $E_{B-V}=$ 0.11. Using $E_{C-T 1}=1.97 E_{B-V}$ (Harris \& Canterna 1977) this means a range in reddening from $E_{C-T 1}=0.16-0.22$. The IRAS map towards this direction is very patchy and thus we decided to use just a mean reddening value of $E_{C-T 1}=0.19$ if not otherwise stated (e.g. for our background comparison field). For the absorption in $R$ we use the relation $A_{R} / A_{V}=0.75$ (Rieke \& Lebofsky 1985).

\subsection{Photometric completeness}

The completeness of the data has been studied with the aid of the task addstar within DAOPhot II, which was used to add 6666 stars to the science image. This was done ten times to produce ten different images. These modified images were reduced in the same way as the original data. The final completeness function for the color range $0.9<(C-T 1)<2.5$ is plotted in Fig. 3 for the whole MOSAIC field. The difference between the completeness function for red and blue clusters is marginal and does not need to be considered. However, there are strong spatial variations: nearer to the center of the elliptical galaxies and in the vicinity of bright stars the completeness is lower. Instead of using a spatial variable completeness function we just excluded areas in which the completeness deviates more than $5 \%$ from its overall value.

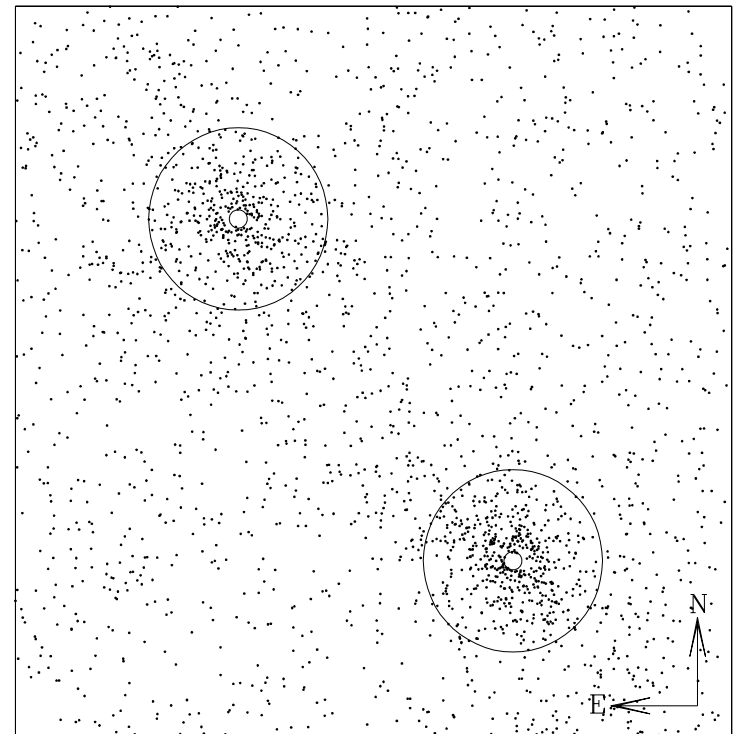

Fig. 4. The spatial distribution of all point sources fainter than $T 1=$ 21.5 within the color range $0.9<(C-T 1)<2.3$ are plotted with the same orientation as Fig. 1. We indicate the areas which were used to derive the color distributions of point sources plotted in Fig. 5.

These completeness calculations also serve to check the reliability of the photometry and the errors given by DAOPhot. No systematic difference between input and output magnitude were found. In the lower panel of Fig. 3, the DAOPhot errors are compared to the standard deviation of the difference between input and output magnitudes of the simulated stars. It can be seen that this standard deviation is larger than the DAOPhot errors and we used it instead as the uncertainty of our measurements.

\section{Properties of the globular cluster systems}

In the Washington color $(C-T 1)$ globular clusters can be found in the range $0.9<(C-T 1)<2.3$ (e.g. Geisler et al. 1996b; Forte et al. 2001; Dirsch et al. 2003). We selected as cluster candidates all point sources within this color range that are fainter than $T 1=21.5$. The spatial distribution of these objects is shown in Fig. 4. The GCSs of the two larger elliptical galaxies - NGC 3258 and NGC 3268 - can be readily seen.

\subsection{Color distributions}

To derive the GC color distribution, all point sources within a radial range of $1^{\prime}<r<4.5$ around NGC 3258 and NGC 3268 (shown in Fig. 4) are selected. Point sources within the GC candidate's color range that are further than $10^{\prime}$ away from the ellipticals and further than $2^{\prime}$ away from any other galaxy serve as a background sample. This background might still be contaminated by globular clusters and we might slightly underestimate the number of globulars in the galaxies

The color distributions of the background corrected cluster candidates are plotted in Fig. 5 where we use the adaptive filter method described by Fadda et al. (1998) that uses an adaptive Epanechnikov kernel (the color distribution is tabulated 

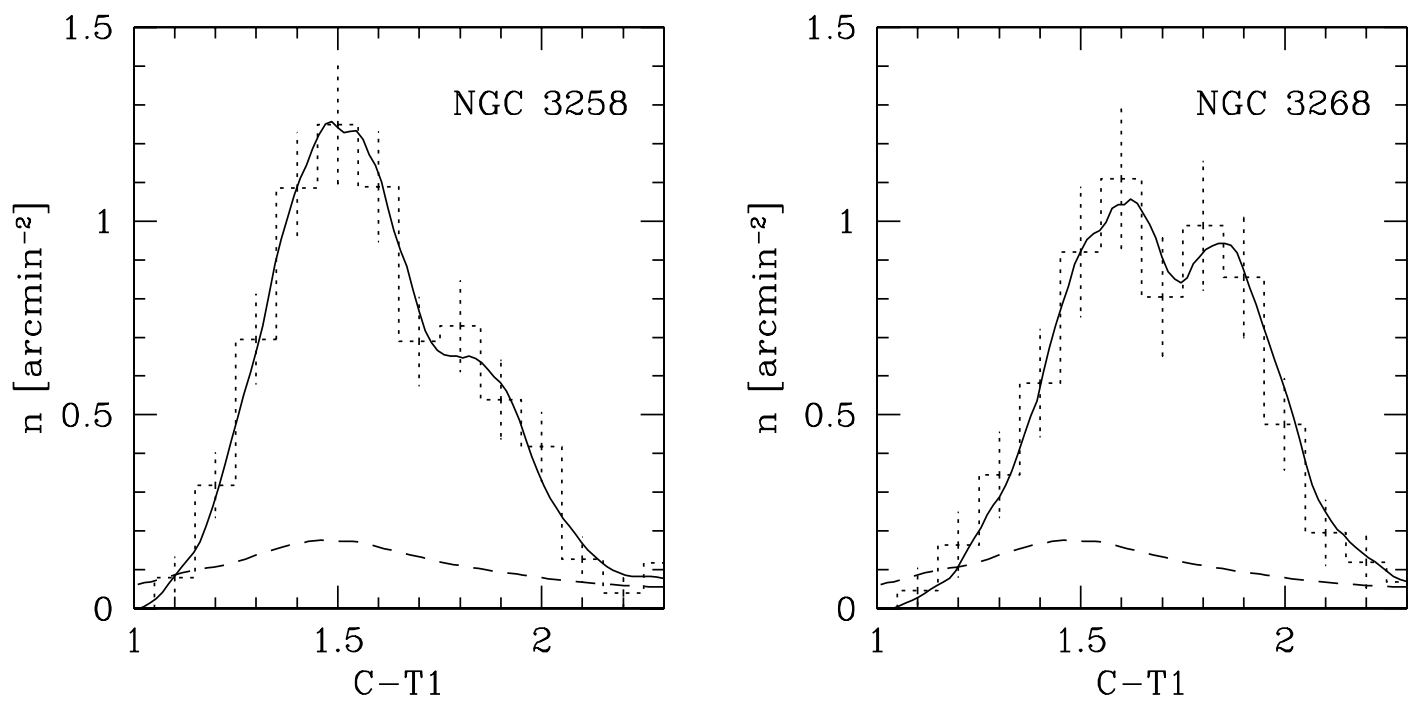

Fig. 5. The color distribution functions for the GC candidates (background corrected) within a radial distance of $4.5^{\prime}$ around NGC 3258 and NGC 3268 are shown as the solid lines. We used an Epanechnikov kernel for the adaptive smoothing. The applied background for the correction is displayed as the dashed line. The dotted line shows the histogram of the data and the errors.

in Table 2). This approach facilitates the visual inspection of the distributions, in particular, it facilitates the identification of peaks and the recognition of the peak positions.

Around NGC 3268 and NGC 3258 the GCSs show up nicely as bimodal color distributions, which is due to the good metallicity resolution of the Washington $C-T 1$ color. The two peaks can be determined by using a phenomenological fit of two Gaussians. We applied the reddening correction from Table 1 and found for the peak positions $C-T 1=1.36 \pm 0.04$ and $C-T 1=1.70 \pm 0.02$ for the GCS of NGC 3258 and $C-T 1=1.31 \pm 0.02$ and $C-T 1=1.66 \pm 0.03$ for NGC 3268 . The widths of the fitted Gaussians for the blue/red peaks were $0.16 \pm 0.02 / 0.17 \pm 0.02$ and $0.15 \pm 0.02 / 0.19 \pm 0.02$ for NGC 3258 and NGC 3268, respectively.

\subsection{Radial distribution}

The radial distributions of the cluster candidates around NGC 3258 and NGC 3268 are plotted in Fig. 6 and tabulated in Table 3. The radial number distribution of the GCS is most frequently described as a power-law and we find, using the clusters within $1^{\prime}$ and $10^{\prime}$, a power-law index of $-1.96 \pm 0.13$ for NGC 3258 and of $-1.81 \pm 0.13$ for NGC 3268 . These fits are indicated in Fig. 6 which shows that in particular for NGC 3258 a single power-law is a rather poor fit. To account for the changing slope we use a function which is motivated by the form of a modified Hubble distribution (Binney \& Tremaine 1987) which includes a core radius $r_{0}$ and which converges to a power-law of index $2 \beta$ at large radii:

$n(r)=a\left(1+\left(\frac{r}{r_{0}}\right)^{2}\right)^{-\beta}$

where $n(r)$ is the surface number density. We found that the following parameters provided the best fit:

- NGC 3258: $a=23 \pm 7, r_{0}=1.7^{\prime} \pm 0.5^{\prime}, \beta=1.3 \pm 0.2$

- NGC 3268: $a=20 \pm 6, r_{0}=1.5^{\prime} \pm 0.5^{\prime}, \beta=1.2 \pm 0.2$.
Table 2. Color distribution for the cluster candidates around NGC 3268 and NGC 3258. A uniform reddening correction of $m C-T 1=0.19$ was applied. All clusters brighter than $R=24$ were taken into account.

\begin{tabular}{|c|c|c|}
\hline$C-T 1$ & $\begin{array}{c}n_{\mathrm{NGC}} 3258 \\
{\left[\operatorname{arcmin}^{-2} 0.1 \mathrm{mag}^{-1}\right]}\end{array}$ & $\begin{array}{c}n_{\mathrm{NGC}} 3268 \\
{\left[\operatorname{arcmin}^{-2} 0.1 \mathrm{mag}^{-1}\right]}\end{array}$ \\
\hline 1.00 & $0.02 \pm 0.01$ & $0.00 \pm 0.01$ \\
\hline 1.10 & $0.11 \pm 0.03$ & $0.04 \pm 0.02$ \\
\hline 1.15 & $0.19 \pm 0.04$ & $0.06 \pm 0.03$ \\
\hline 1.20 & $0.33 \pm 0.07$ & $0.12 \pm 0.04$ \\
\hline 1.25 & $0.57 \pm 0.11$ & $0.21 \pm 0.05$ \\
\hline 1.30 & $0.80 \pm 0.14$ & $0.30 \pm 0.07$ \\
\hline 1.35 & $1.05 \pm 0.17$ & $0.45 \pm 0.10$ \\
\hline 1.40 & $1.22 \pm 0.19$ & $0.63 \pm 0.12$ \\
\hline 1.45 & $1.36 \pm 0.21$ & $0.86 \pm 0.15$ \\
\hline 1.50 & $1.40 \pm 0.22$ & $0.99 \pm 0.19$ \\
\hline 1.55 & $1.35 \pm 0.22$ & $1.14 \pm 0.22$ \\
\hline 1.60 & $1.20 \pm 0.21$ & $1.17 \pm 0.24$ \\
\hline 1.65 & $1.02 \pm 0.18$ & $1.09 \pm 0.23$ \\
\hline 1.70 & $0.87 \pm 0.16$ & $1.00 \pm 0.20$ \\
\hline 1.75 & $0.77 \pm 0.14$ & $0.94 \pm 0.18$ \\
\hline 1.80 & $0.73 \pm 0.14$ & $0.92 \pm 0.16$ \\
\hline 1.85 & $0.72 \pm 0.15$ & $0.97 \pm 0.16$ \\
\hline 1.90 & $0.67 \pm 0.15$ & $0.95 \pm 0.17$ \\
\hline 1.95 & $0.57 \pm 0.13$ & $0.85 \pm 0.16$ \\
\hline 2.00 & $0.40 \pm 0.10$ & $0.68 \pm 0.14$ \\
\hline 2.05 & $0.29 \pm 0.08$ & $0.54 \pm 0.12$ \\
\hline 2.10 & $0.21 \pm 0.06$ & $0.39 \pm 0.09$ \\
\hline 2.15 & $0.15 \pm 0.05$ & $0.30 \pm 0.07$ \\
\hline 2.20 & $0.11 \pm 0.03$ & $0.24 \pm 0.06$ \\
\hline 2.25 & $0.07 \pm 0.02$ & $0.17 \pm 0.05$ \\
\hline 2.30 & $0.07 \pm 0.02$ & $0.12 \pm 0.04$ \\
\hline
\end{tabular}



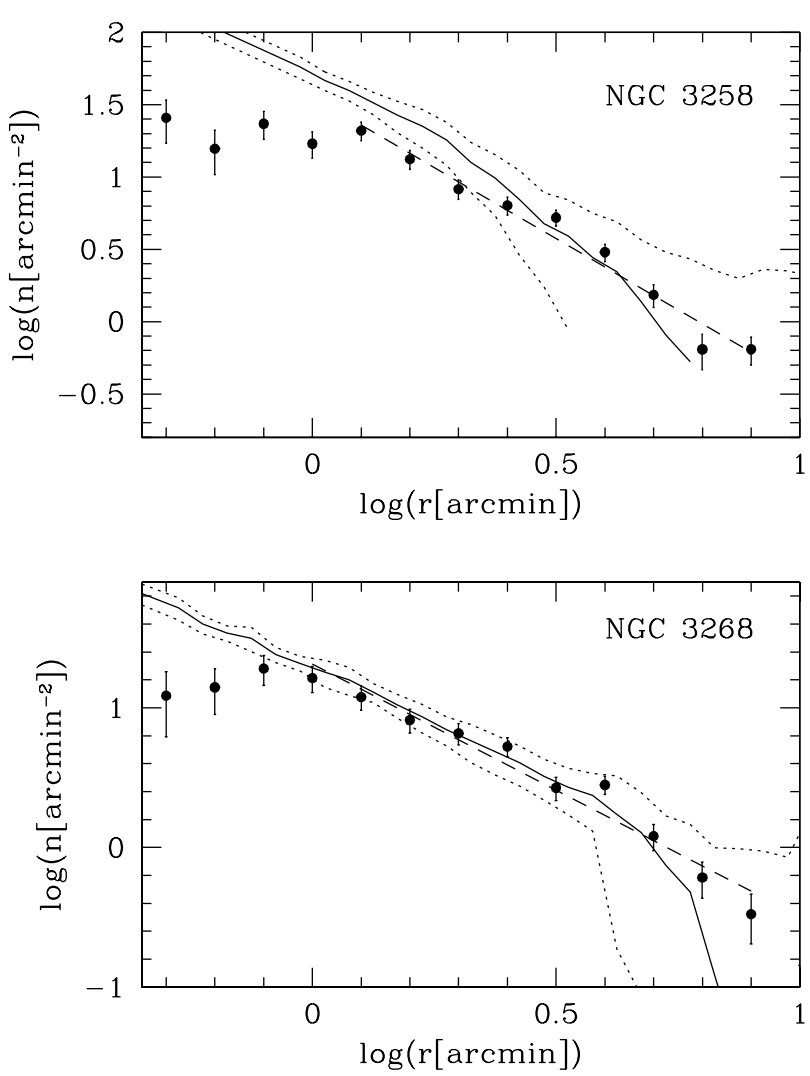

Fig. 6. This graph shows the radial distribution for the GC candidates $(0.9<(C-T 1)<2.3,21.5<T 1<23)$ for NGC 3258 and NGC 3268. Within 0.7 the measurements are affected by radial incompleteness effects. The dashed lines show the fit to the data described in the text. The galaxy T1 luminosity profile multiplied by -0.4 and arbitrarily shifted is shown as the solid line with $1 \sigma$ uncertainties (dotted lines). The galaxy measurements are described in Sect. 4.

It is neither possible to detect any difference in the distribution of red and blue clusters, as frequently seen in other elliptical galaxies (e.g. NGC 1399, Dirsch et al. 2003; NGC 4472, Rhode \& Zepf 2001), nor to separate the GCS into two populations with different mean colors and different radial distributions. Perhaps these effects are blurred by small number statistics and deeper observations are needed to clarify this point.

\subsection{Azimuthal distribution}

The azimuthal number densities of the GCSs of NGC 3258 and NGC 3268 within a radial range 1.'6 $<r<4.5$ are shown in Fig. 7. The azimuthal angle is defined as a position angle, measured from North to East. An elliptical GCS causes sinusoidal counts in this diagram with the ellipticity $\epsilon=1-\left(N_{b} / N_{a}\right)^{1 / \alpha}$ ( $N_{b}, N_{a}$ being the number of clusters along minor and major axis respectively and $\alpha$ the absolute value of the exponent of the radial distribution).

The GCS of NGC 3258 is clearly elongated and a sinusoidal fit (also shown in Fig. 7) yielded an ellipticity of $\epsilon=0.26 \pm 0.09$ and a position angle of $38^{\circ} \pm 6^{\circ}$. Also the GCS of NGC 3268 is elongated, but it has a lower ellipticity of $\epsilon=0.15 \pm 0.09$. Its position angle has been determined to be at $47^{\circ} \pm 9^{\circ}$.
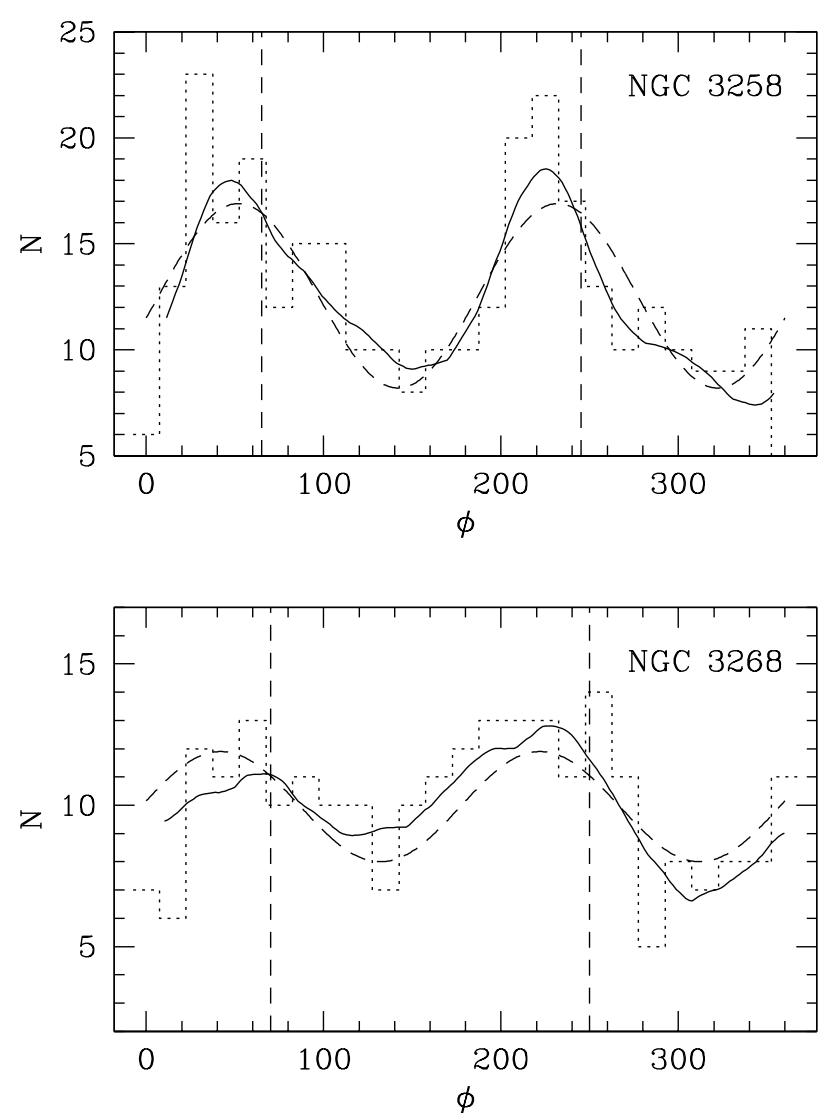

Fig. 7. The azimuthal distribution of the globular clusters within a radial distance of 1.' $6<r<4.5$ around NGC 3258 and NGC 3268. The dotted line shows the binned azimuthal distribution and the solid line the smoothed one that was also used for a $\sin ^{2}$-fit shown as the dashed line. This graph shows that both GCSs have an elliptical shape. The vertical dashed lines show the position angle of the galaxy light (see Sect. 4).

\subsection{The total number of GCs}

The total number of clusters can of course not be measured directly, yet it is well known that the luminosity function of GCs can be described (in magnitudes) with a Gaussian that has a characteristic turn-over magnitude (TOM) and a limited range of possible widths. Assuming a distance towards the two elliptical galaxies, a TOM and a width of the Gaussian, we can extrapolate the number of observed GCs to fainter magnitudes covering the whole luminosity function. We assumed a TOM for the Milky Way GCS of $V=-7.61 \pm 0.08$ and a metallicity correction derived by Ashman et al. (1995) of 0.2 (corresponding to a mean cluster metallicity of -0.6 dex, adequate for the two elliptical galaxies). As we do not have $V$ observations, we have to assume a $V-R$ color of $V-R=0.6$ (taken from the Milky Way clusters of a similar metallicity). Thus, we expect the TOM to be at $M_{\mathrm{TOM}}=-8.0$ in $R$ and hence an unreddened $m_{\mathrm{TOM}}=24.7 \pm 0.25$. This value can also be used for $T 1$ since there is only a negligible zero point difference between $T 1$ and $R$ with very little color dependence. For the width of the Gaussian, values from 1.2 to 1.4 are typically found in elliptical galaxies of this size in the literature (Ashman \& Zepf 1998 and references therein). 
Table 3. Radial cluster density distribution $(0.9<(C-T 1)<2.3$, $21.5<T 1<23$ ) for NGC 3268 and NGC 3258.

\begin{tabular}{ccc}
\hline \hline $\begin{array}{c}\log (r) \\
{[\operatorname{arcmin}]}\end{array}$ & $\begin{array}{c}\log \left(n_{\mathrm{NGC} 3268}\right) \\
{\left[\operatorname{arcmin}^{-2}\right]}\end{array}$ & $\begin{array}{c}\log \left(n_{\mathrm{NGC} 3258}\right) \\
{\left[\operatorname{arcmin}^{-2}\right]}\end{array}$ \\
\hline-0.3 & $1.41_{-0.17}^{+0.12}$ & $1.09_{-0.29}^{+0.17}$ \\
-0.2 & $1.20_{-0.18}^{+0.13}$ & $1.15_{-0.20}^{+0.13}$ \\
-0.1 & $1.37_{-0.11}^{+0.09}$ & $1.28_{-0.12}^{+0.09}$ \\
0.0 & $1.23_{-0.10}^{+0.08}$ & $1.21_{-0.10}^{+0.08}$ \\
0.1 & $1.32_{-0.07}^{+0.06}$ & $1.08_{-0.10}^{+0.08}$ \\
0.2 & $1.12_{-0.07}^{+0.06}$ & $0.91_{-0.09}^{+0.08}$ \\
0.3 & $0.92_{-0.07}^{+0.06}$ & $0.82_{-0.08}^{+0.07}$ \\
0.4 & $0.80_{-0.07}^{+0.06}$ & $0.72_{-0.07}^{+0.06}$ \\
0.5 & $0.72_{-0.06}^{+0.05}$ & $0.43_{-0.09}^{+0.08}$ \\
0.6 & $0.48_{-0.07}^{+0.06}$ & $0.45_{-0.07}^{+0.06}$ \\
0.7 & $0.18_{-0.09}^{+0.07}$ & $0.08_{-0.10}^{+0.08}$ \\
0.8 & $-0.19_{-0.14}^{+0.11}$ & $-0.22_{-0.15}^{+0.11}$ \\
0.9 & $-0.19_{-0.11}^{+0.09}$ & $-0.48_{-0.21}^{+0.14}$ \\
\hline
\end{tabular}

We used clusters within a radial distance of $4.5^{\prime}$ to construct the globular cluster luminosity functions (GCLF) that are shown in Fig. 8. The GCLF of NGC 3268 is peculiar: it shows an enhancement of point sources around $22.4<T 1<22.6$. These objects are not spatially concentrated in any particular way. Also the apparent deficiency of point sources fainter than $T 1=23.4$ is striking, since we cannot have reached the TOM at such bright magnitudes. The reason might be an underestimation of the completeness at these magnitudes, since in the vicinity of NGC 3268 there are 4 brighter spiral galaxies. However, in the following we take only clusters brighter than 23.5 into account and considering the other uncertainties mentioned below, we do not regard this as a severe problem. Further observations are nevertheless required for a deeper and more complete study of this peculiar luminosity function.

Since we observe only $\approx 10 \%$ of all clusters around the galaxies, changes in the distance and the width of the Gaussian can result in large uncertainties in the total number of GCs. We find for the total number of GCs, within a radial distance of 4.5, around NGC 3258: $3400 \pm 280_{-1200-600}^{+3600+900}$, and around NGC 3268: $2500 \pm 220_{-900-500}^{+2600+800}$. The first uncertainty is the statistical uncertainty, the second is due to the distance uncertainty, and the third is due to the uncertainty in the width of the distribution. The large uncertainties due to the distance uncertainty and the width of the distribution, do not allow a firmer statement than that the GCSs of both galaxies encompass several thousands GCs and that NGC 3258 contains more clusters within 4.5 (corresponding to $5 r_{\text {eff }}$ and $3.3 r_{\text {eff }}$ for NGC 3258 and NGC 3268, respectively) than NGC 3268.

\section{Properties of the host galaxies}

\subsection{Galaxy luminosity profiles}

We determined the luminosity profiles of NGC 3258 and NGC 3268 with the purpose of comparing them with their GCSs. The longer exposures were used for the outer part (radial distances larger than $30^{\prime \prime}$ ) and the short one for the inner part. Within 2'.5, the task ellipse within IRAF can be used to
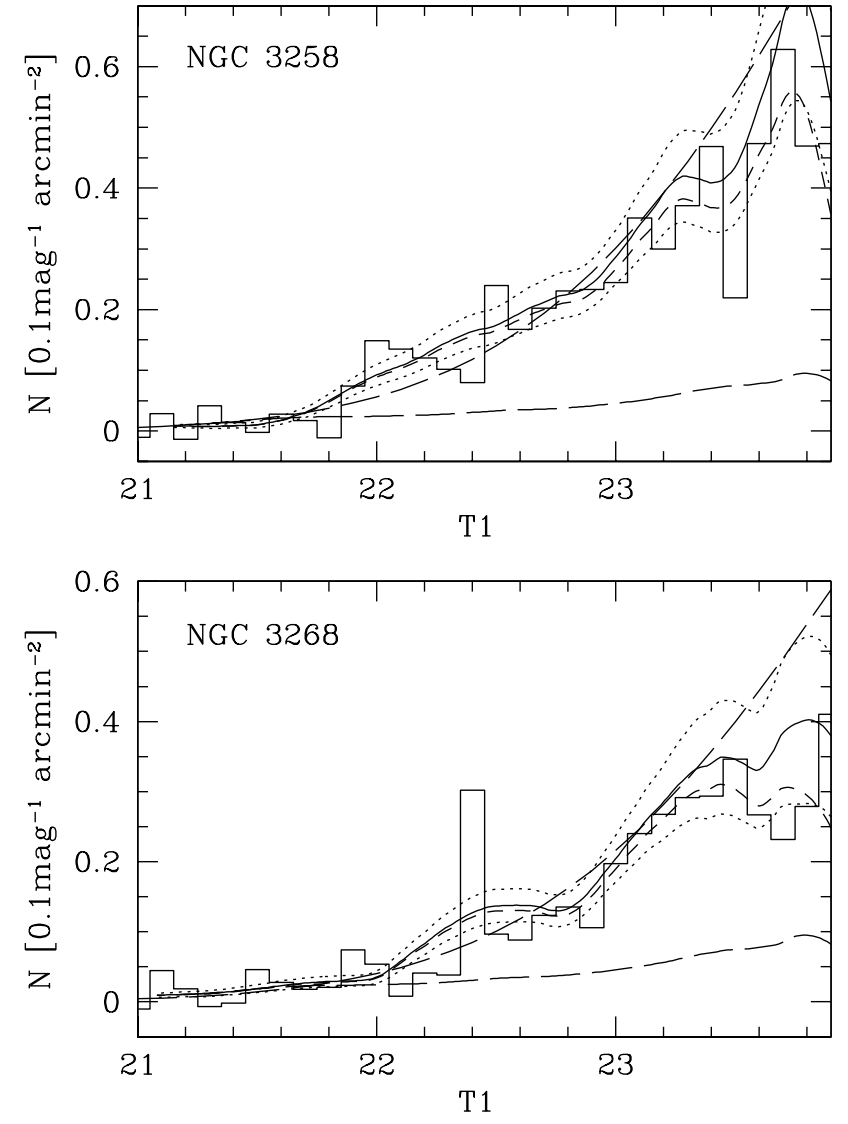

Fig. 8. The completeness corrected luminosity function of GC candidates $(1<(C-T 1)<2)$ with a radial distance between $0.46^{\prime}<r<$ $4.5^{\prime}$ is plotted as histogram and adaptively filtered distribution with a solid line for NGC 3258 in the upper panel and for NGC 3268 in the lower panel. The short dashed line shows the luminosity function before the completeness correction. The lower long dashed lines in the panels indicate the background luminosity function and the upper long dashed line the fitted Gaussian described in the text.

determine the profile; outside, however, the results obtained with ellipse were not satisfactory because of the many disturbing objects and thus we decided to use a similar approach as in Dirsch et al. (2003): we measured the luminosity in 3 pix apertures in 33333 randomly distributed areas around NGC 3258 and NGC 3268 on the point source subtracted images. We then excluded areas that are influenced by galaxies, strong stellar residuals and saturated stars. The disadvantage of this approach is that it is not straightforward to determine the ellipticity and thus this has been done only in the inner 2.5.

The luminosity profiles are plotted in Fig. 9. For comparison, we included in this figure the luminosity profiles of the central Fornax cluster galaxy NGC 1399 (taken from Dirsch et al. 2003). For this comparison we shifted the Fornax galaxy to the Antlia distance. In the inner part $\left(r<3.5^{\prime \prime}\right)$, the profiles of the Antlia galaxies appear flatter than that of NGC 1399. The reason is that HST observations of Lauer et al. (1995) were used to determine the luminosity profile in the inner part of NGC 1399, where the PSF is much smaller than for our data. In Fig. 9 we included the $V$ profiles determined by Reid et al. (1994) for NGC 3268 and NGC 3258. The agreement 

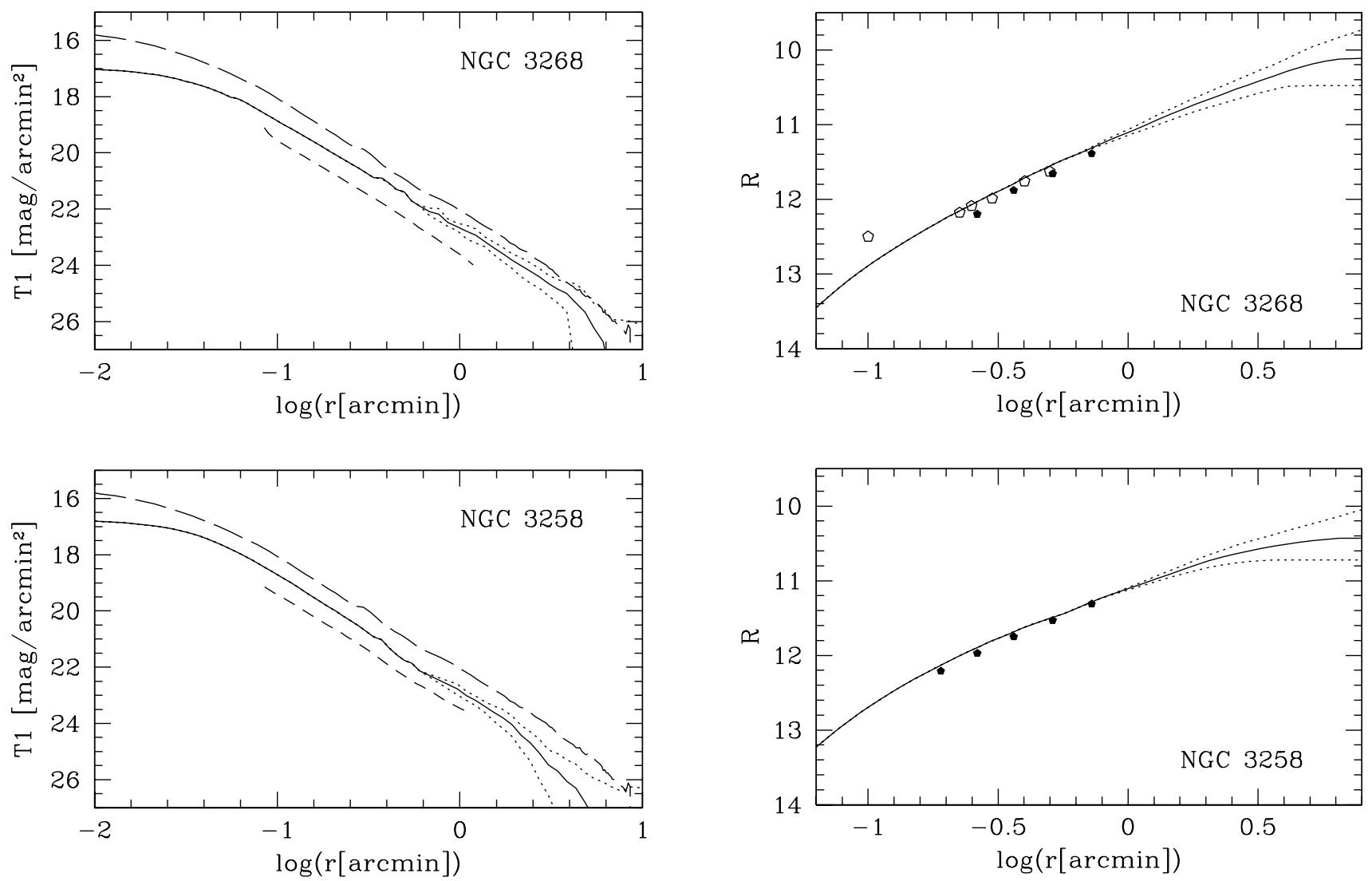

Fig. 9. The luminosity profiles of NGC 3268 and NGC 3258 (solid curves) and $1 \sigma$ uncertainties (dotted line) are compared to the luminosity profile of NGC 1399 (long dashed line). For this comparison the diameter of NGC 1399 has been shrunk by a factor of three to account for the distance difference (a distance modulus of $(m-M)=31.4$ is assumed for NGC 1399). The short dashed lines are the $V$ profiles for NGC 3268 and NGC 3258 published by Reid et al. (1994).

is excellent and a mean $V-R$ color of $V-R \approx 0.7$ can be determined. This color agrees with the value given by Godwin et al. (1977) for NGC 3268.

The luminosity profiles for radii smaller than $5^{\prime}$ can be fitted with the same function as used for the cluster density distribution:

$T 1=a \log \left(1+\left(r / r_{0}\right)^{2}\right)+T 1_{0}$.

The following values have been found:

- NGC 3258: $a=1.99 \pm 0.01, r_{0}=0.036^{\prime} \pm 0.001^{\prime}, T 1_{0}=$ $16.77 \pm 0.01$

- NGC 3268: $a=2.34 \pm 0.02, r_{0}=0.044^{\prime} \pm 0.001^{\prime}, T 1_{0}=$ $16.57 \pm 0.03$.

In Fig. 10 the integrated luminosity in $R$ is shown (we transformed our T1 measurements into $R$ using the tiny shift mentioned in Sect.2). Poulain et al. (1994) determined the luminosity of NGC 3258 within five apertures and their results are shown in Fig. 10 as well. For NGC 3268, aperture photometry has been performed by Godwin et al. (1977) and Poulain et al. (1994), and their measurements are also displayed in Fig. 10.

By means of the $R$ integrated luminosity profiles it is also possible to perform a rough estimation of the effective

radii $\left(r_{\text {eff }}\right)$ of NGC 3258 and NGC 3268 , assuming that the values obtained at the radius of $5^{\prime}$ (about $50 \mathrm{kpc}$ ) represent the total integrated luminosities. Thus, the measured effective radii result are $(8 \pm 4) \mathrm{kpc}$ for NGC 3258 and $(12 \pm 5) \mathrm{kpc}$ for NGC 3268, where the large errors are a consequence of the uncertainties in the luminosity profiles at large radii.

\subsection{Galaxy color profile}

The $\mathrm{C}$ luminosity profiles are used together with the $T 1$ profiles to search for possible color gradients, which is shown in Fig. 11 for the dereddened colors. In this figure it is apparent that both galaxies become bluer with increasing radius. However, the color profiles of both galaxies are quite different at radial distances smaller than $3^{\prime \prime}$. Within this radius NGC 3268 becomes remarkably red. Visual inspection of the $\mathrm{C}$ image showed a clearly off-centered central peak. Both observations can be explained by an inclined circumnuclear dusty disk with a radius of roughly $3^{\prime \prime}$ (which corresponds to $500 \mathrm{pc}$ ).

The observed color gradient of NGC 3258 is in accordance with the $B-V$ gradient found by Reid et al. (1994). However, they found no color gradient in NGC 3268. This might be explained by the fact that the color gradient of this galaxy is not 

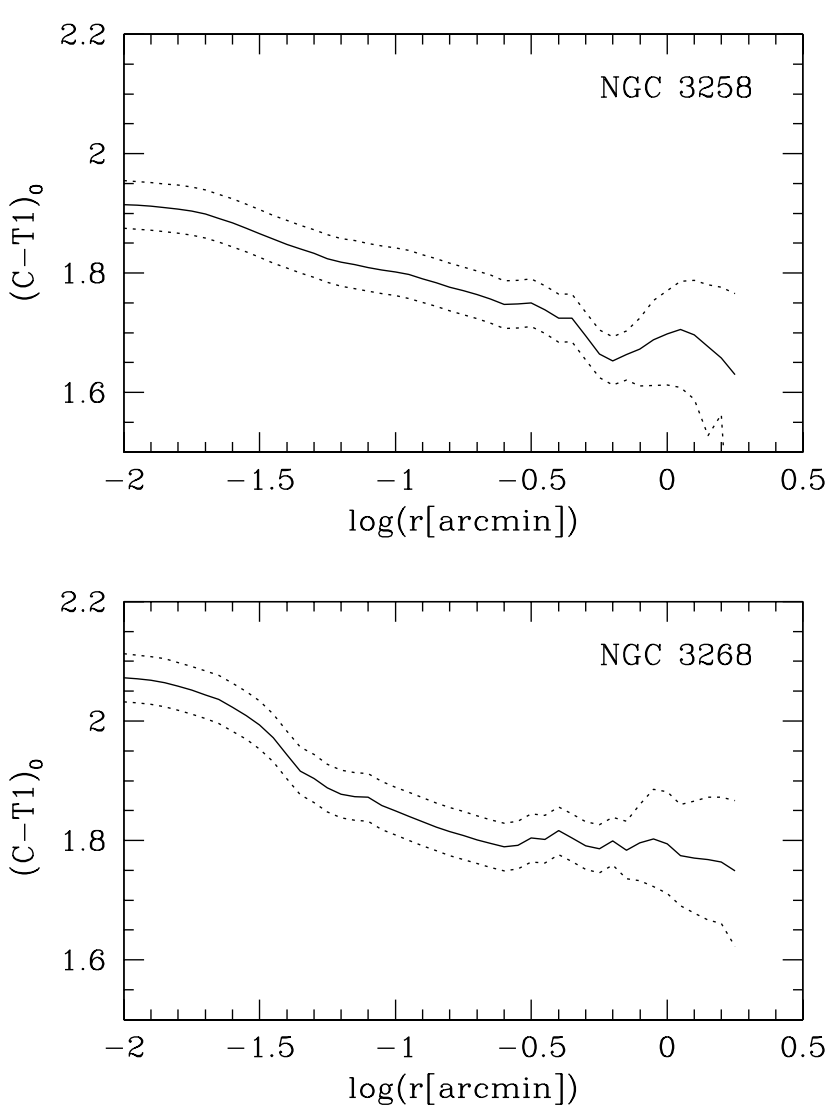

Fig. 11. The color profiles of the two ellipticals are shown together with the $1 \sigma$ uncertainties.

as pronounced as in NGC 3258 at larger radii. In $(V-I)$ no color gradient is apparent for either galaxy (Reid et al. 1994).

\subsection{Galaxy ellipticities}

The ellipticities have been measured within the inner 2.5 using the task ellipse. For NGC 3268, the derived ellipticity agrees well within 1' with the values derived by Reid et al. (1994); further outside, however, the ellipticity of Reid et al. increases while in our model it remains constant at $\epsilon=0.20 \pm 0.08$ out to 2 '.5. The position angle of $\approx 70^{\circ}$ of Reid et al. (1994) agrees with our value of $70^{\circ} \pm 5$ up to 2.5 (the uncertainties were derived by varying initial conditions and fit parameters of the task ellipse). Also for NGC 3258, our results confirm the earlier measurements by Reid et al. (1994): the ellipticity increases from 0.1 to about 0.3 at 2.5 . The position angle decreases in the same radial range from $75^{\circ} \pm 5^{\circ}$ to $65^{\circ} \pm 5^{\circ}$.

The azimuthal distributions of the GCSs, presented in Sect. 3.3, are estimated within the radial range 1.'6 $<r<4.5$, that is, partially overlapping the area over which the galaxies' ellipticities have been calculated $(r<2$ '5). We cannot strictly compare the GCS and the galaxy light within the same radial range, since at small radii the incompleteness does not allow reliable determination of the GCS ellipticity and at radii larger than 2.5 the ellipticity of the galaxy cannot be determined due to the low surface brightness and the accompanying galaxies.

\subsection{Comparing the light profiles of NGC $3268 / 3258$ and NGC 1399}

It is interesting to compare the luminosity profiles of NGC 3268 and NGC 3258 with the profile of the central Fornax cluster galaxy NGC 1399 (Fig. 9). This comparison shows that the luminosity profile of NGC 3268 resembles that of NGC 1399 very closely. The luminosity profile of NGC 3258 seems to be steeper than that of NGC 1399 at radii larger than $2^{\prime}$. However, within the uncertainties it is also consistent with having no change in the slope. Better data is needed for an interpretation of its profile.

The surface luminosity of both galaxies is considerably lower that that of NGC 1399 (projected to the same distance), the reason being foreground reddening which accounts for an absorption in $R$ of 0.26 for NGC 3268 and 0.19 for NGC 3258 .

The deprojected luminosity density of NGC 3268 thus is close to a $r^{-3}$ profile as in the case of NGC 1399 (Dirsch et al. 2003) with a decline to a steeper profile only at very large radii. NGC 3258 shows a steeper profile at clearly smaller radii. The transition from a $r^{-3}$ to a $r^{-4}$ density profile, which in projection gives the familiar de Vaucouleurs profile, is probably caused by a sharp cut in the energy distribution function near the escape energy in the case of an approximate Keplerian potential, as has been argued by Jaffe (1987) and White (1987). In this line of argument Dirsch et al. (2003) attributed the $r^{-3}$ decline (deprojected) of NGC 1399 to the presence of its strong dark matter potential. In analogy also NGC 3268 may be located inside a deep potential of dark matter that constitutes the Antlia cluster, which is consistent with the results from the $\mathrm{X}$-ray observations.

\section{Comparing the GCS and the galaxy light}

\subsection{Specific frequency}

The specific frequency is the number of clusters $(N)$ normalized to the $V$ luminosity of the galaxy $\left(S_{N}=N \times 10^{0.4\left(M_{V}+15\right)}\right)$. Hence the total luminosity of the galaxy is required.

In our case the large luminosity uncertainties at $8^{\prime}$, our outermost radius, do not permit us to derive a reliable global specific frequency. To circumvent this problem we consider only the circular area with a radius of $4^{\prime}$ for the calculation of the specific frequency, which corresponds to about $40 \mathrm{kpc}$ at the distance of the Antlia cluster, or approximately $5 r_{\text {eff }}$ for NGC 3258 and $3.3 r_{\text {eff }}$ for NGC 3268, according to the rough values obtained in Sect. 4.1. This is the largest radius for which a reasonably accurate total luminosity can be obtained (see Fig. 10). The large uncertainty of the absolute magnitude and of the total number of clusters due to the distance uncertainty is not too critical, since these uncertainties are coupled - i.e. a larger distance means a brighter galaxy, but also more globular clusters. We used a color of $V-R=0.7$ for NGC 3268 (Godwin et al. 1977, slightly redder than the color used for the GCs above, $V-R=0.6)$. For the reddening correction the relation given by Rieke \& Lebofsky (1985), $E(V-R) / E(B-V)=$ 0.78 is used. The luminosity within $4^{\prime}$ is $R=10.22 \pm 0.15$ and $R=10.45 \pm 0.18$ for NGC 3268 and NGC 3258, respectively. 
Using our distance modulus and the reddening values from Table 1 we obtain for the absolute luminosity of NGC 3268 and NGC 3258, $M_{R}=-22.77 \pm 0.30$ and $M_{R}=-22.10 \pm 0.31$, respectively. With $V-R=0.7$, we finally find a specific frequency of $S_{N}=3.0 \pm 2.0$ for NGC 3268 and of $S_{N}=6.0 \pm 2.6$ for NGC 3258 (the mean value is calculated for a width of the Gaussian GCLF of 1.3 and a distance of $(m-M)=32.73)$.

\subsection{Morphological comparison}

In Fig. 6 the luminosity profiles are compared to the radial cluster distributions. For NGC 3268, both radial profiles agree very well for radii larger than 0 '. $^{\prime}$. At smaller radii the clusters have a shallower distribution, which predominately is caused by the radial incompleteness variations. When only brighter clusters are used, the slope in the inner region increases; however, the noise also increases due to the smaller number statistics. In the outer region, the slope remains independent from the choice of the lower limiting magnitude within the uncertainties. In NGC 3258 the large uncertainties of the luminosity profile in the overlapping area make a statement more difficult. Within the uncertainties, the GC density distribution and luminosity profile agree, albeit there is an indication that the clusters have a slightly shallower distribution than the $T 1$ profile. Such a behavior is expected in a galaxy in which a metal poor cluster population has a shallower distribution than a more metal rich one, since the galaxy luminosity profile is measured in a relatively red passband. Since clusters are accompanied by field populations, the different distribution of the two cluster populations should be reflected in the field populations. This picture agrees well with the fact that NGC 3258 shows a color gradient. Nevertheless, as stated above, we were unable to disentangle the GCS into two populations that have a different mean color and a different radial distribution. However, this might be due to small number statistics and further observations are required to clarify this strange behavior.

The cluster systems and the galaxies are elliptical with similar ellipticities at overlapping radii. NGC 3258 becomes progressively more elliptical with radius, which is also reflected in its rather elongated GCS, while NGC 3268 maintains approximately its shape. The position angles deviate slightly between galaxy light and cluster system in both galaxies. This deviation might, however, be understandable for NGC 3258 where the position angle of the galaxy light monotonically changes with radius and, extrapolating it to the GCS radial regime, we expect the observed GCS position angle also for the galaxy light.

It is noteworthy that the position angles of the GCS in both galaxies are approximately aligned with the connecting line of the two galaxies that has a position angle of $50^{\circ}$.

\section{Summary}

This investigation demonstrated the presence of a few thousand GCs in NGC 3258 and NGC 3268. The resulting specific frequencies are $S_{N}=3.0 \pm 2.0$ for NGC 3268 and $S_{N}=$ $6.0 \pm 2.6$ for NGC 3258. These specific frequencies were calculated within a radius of $40 \mathrm{kpc}$, which corresponds to approximately $5 r_{\text {eff }}$ for NGC 3258 and $3.3 r_{\text {eff }}$ for NGC 3268, as said above. The specific frequency of NGC 3268 is typical for an elliptical galaxy (e.g. Ashman \& Zepf 1998), while the one of NGC 3258 is comparable to giant ellipticals in the center of galaxy clusters, like NGC 1399 (Dirsch et al. 2003). However, given the uncertainties, these values should be regarded as being indicative only.

The color of the red and blue peaks of the GCS appear to be very similar to that of other galaxies that were studied in the Washington system (NGC 1399 - Dirsch et al. 2003; NGC 4472 - Geisler et al. 1996b; M 87 - Côté et al. 2001; NGC 1427 - Forte et al. 2001). This was expected given that investigations in $V$ and $I$ also resulted in similar, if not equal colors (e.g. Kundu \& Whitmore 2001a; Forbes \& Forte 2001a; Larsen et al. 2001; Gebhardt \& Kissler-Patig 1999).

The luminosity profile of NGC 3268 resembles that of NGC 1399 remarkably well.

We compared the spatial distribution of the stellar light and the GCS and found (at least in the outer range $-r>1.7^{\prime}$ ) the same profiles. Differences in the distribution of red and blue subpopulations are not discernible due to poor number statistics. Furthermore, the major axes of both galaxies are within the uncertainties aligned with their connecting axis, resembling the X-ray findings. Whether this is due to a physical process or whether it is a chance alignment can not be answered by this study.

\section{Appendix A: A strange absorption feature in NGC 3269}

During the visual inspection of the frames we noted a compact absorption feature in NGC 3269, a spiral galaxy that has no noticeable GCS. The dust cloud can be seen in the southwestern part as shown in a C image (Fig. A.1). NGC 3269 is strikingly devoid of gas (Barnes et al. 2001) and exhibits a very smooth structure. The absorption feature has a diameter of $4^{\prime \prime}$ and can also been seen on previous images of NGC 3269, for example in the Sandage \& Betke (1994) atlas. At the distance of NGC 3269 it would have a diameter of approximately $500 \mathrm{pc}$. It is an intriguing question whether this cloud is indeed in NGC 3269 or whether it is a foreground object in the Galaxy. One approach for further insight is to study its reddening law.

One can estimate the reddening law assuming that the obscured part is similar to the adjacent surface luminosity of the galaxy, which is a reasonable assumption considering its smooth appearance. We obtained by linear interpolation between adjacent regions an absorption of $A_{C}=0.62 \pm 0.08$ and $A_{R}=0.29 \pm 0.08$. In analogy to the absorption in $V$, we define $R_{R}=\frac{A_{R}}{E_{C-R}}=0.9 \pm 0.3$. Using the relations of Harris \& Canterna (1977) and $R_{V}=\frac{A_{V}}{E_{B-V}}=3.2$ we would expect $R_{R}=\frac{0.75 A_{V}}{1.97 E_{B-V}}=1.22$. The main result is that the derived $R$ value is not larger than the expected value which would imply a grayer reddening law.

The singular existence of a dusty region of $500 \mathrm{pc}$ diameter in a galaxy which otherwise is devoid of gas and dust is by itself peculiar. Moreover, it should have substructure leading to small-scale opacity variations for different lines of sight. Unless it is located well above the plane of NGC 3269 (which 


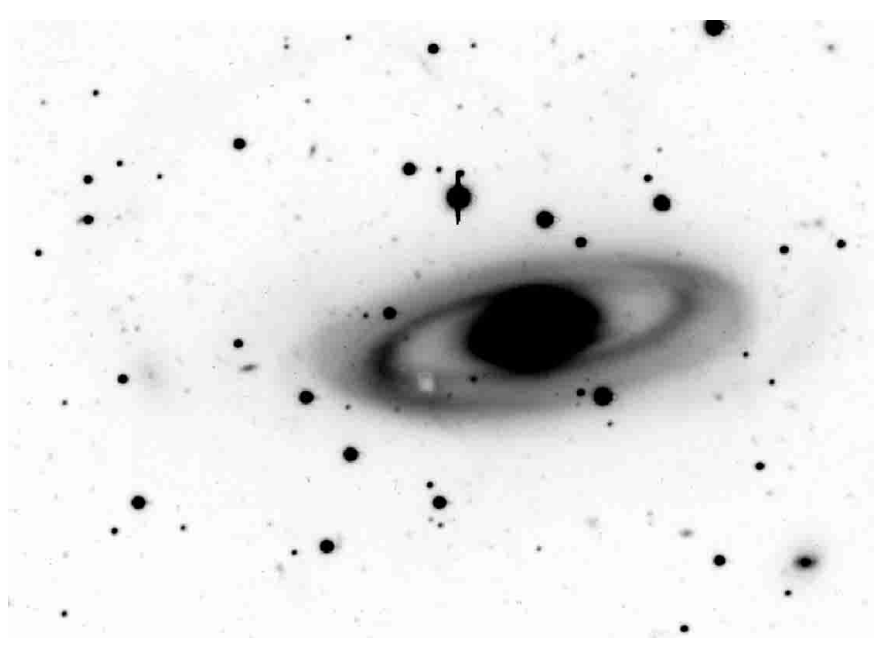

Fig. A.1. $C$ image of NGC 3269 that shows the compact absorption feature in the spiral arm to the lower left. North is to the right, East to the top. The size of the image is $4.9^{\prime} \times 3.7^{\prime}$.

would be another peculiar feature) we expect light contribution from the foreground stellar population. Both facts would result in a grayer reddening law, i.e. a higher $R$ value. Thus the observation of a rather lower $R$ value is an indication that this cloud indeed is located within our galaxy. To give an idea of the possible cloud size in this case, we might estimate an upper limit of its size by assuming a scale height of the galactic disk of $800 \mathrm{pc}$ (which is certainly rather large); the diameter would be less than $0.06 \mathrm{pc}$. Dust clouds with these properties should be extremely difficult to detect, unless they are by chance projected onto a suitable background source.

It could be the optical detection of the same type of compact Galactic gas clouds reported by Heithausen (2002). He found two molecular structures smaller than $1^{\prime}$ (one being $\leq 25^{\prime \prime}$ in CO). The absorption was estimated to be $A_{V} \leq 0.2$ via the HI column density, which is of the same order as our determined absorption $\left(A_{V}=0.4\right)$.

Acknowledgements. TR and BD gratefully acknowledge support from the Chilean Center for Astrophysics FONDAP No. 15010003. LB is also thankful to the Astronomy Group in Concepcion for the financial support and warm hospitality. BD gratefully acknowledges financial support of the Alexander von Humboldt Foundation via a Feodor Lynen Stipendium and spiritual support in the "30 y tantos". We thank an anonymous referee for her/his helpful comments.

\section{References}

Ashman, K. M., \& Zepf, S. E. 1992, ApJ, 384, 50

Ashman, K. M., Conti, A., \& Zepf, S. E. 1995, AJ, 110, 1164

Ashman, K. M., \& Zepf, S. E. 1998, Globular Cluster Systems, Cambridge Astrophysics Series (Cambridge: Cambridge University Press)

Barnes, D. G., \& Webster, R. L. 2001, MNRAS, 324, 859

Beasley, M. A., Baugh, C. M., Ducan, A. F., et al. 2002, MNRAS, 333, 383
Beasley, M. A., Harris, W .E., Harris, G. L. H., \& Forbes, D. A. 2003, MNRAS, 340, 341

Binney, J., \& Tremaine, S. 1987, Galactic Dynamics (Princeton University Press)

Burstein, D., \& Heiles, C. 1982, AJ, 87, 1165

Côté, P., Mc Laughlin, D. E., Hanes, D. A., et al. 2001, ApJ, 559, 828

Côté, P., West, M. J., \& Marzke, R. O. 2002, ApJ, 567, 853

Dirsch, B., Richtler, T., Geisler, T., et al. 2003, AJ, 125, 1908

Fadda, D., Slezak, E., \& Bijaoui, A. 1998, A\&AS, 127, 335

Ferguson, H. C., \& Sandage, A. 1990, AJ, 100, 1

Ferguson, H. C., \& Sandage, A. 1991, AJ, 101, 765

Forbes, D. A., \& Forte, J. C. 2001, MNRAS, 322, 257

Forbes, D. A., Brodie, J. P., \& Grillmair, C. J. 1997, AJ, 113, 1652

Forte, J. C., Geisler, D., Ostrov, P. G., et al. 2001, AJ, 121, 1992

Gebhardt, K., \& Kissler-Patig, M. 1999, AJ, 118, 1526

Geisler, D. 1996, AJ, 111, 480

Geisler, D., Lee, M. G., \& Kim, E. 1996, AJ, 111, 1529

Godwin, J. G., Bucknell, M. J., Dixon, K. L., et al. 1977, Observatory 97, 238

Harris, H. C., \& Canterna, R. 1977, AJ, 82, 798

Harris, W. E. 2001, Star Clusters, Saas-Fee Advanced Course 28, Lecture Notes in Physics, 1998, ed. L. Labhardt, \& B. Binggeli (Berlin: Springer-Verlag)

Harris, W. E., Kavelaars, J. J., Hanes, D. A., Hesser, J. E., \& Pritchet, C. J. 2000, ApJ, 533, 137

Heithausen, A. 2002, A\&A, 393, L41

Hopp, U., \& Materne, J. 1985, A\&AS, 61, 93

Ikebe, Y., Ezawa, H., Fukazawa, Y., et al. 1996, Nature, 379, 427

Ikebe, Y., Makishima, K., Ezawa, H., et al. 1997, ApJ, 481, 660

Jaffe, W. 1987, in Structure and Dynamics of Elliptical Galaxies, ed. T. de Zeeuw (Dordrecht: D. Reidel), IAU Symp., 127, 511

Jones, C., Stern, C., Forman, W., et al. 1997, ApJ, 482, 143

Kundu, A., \& Whitmore, B. C. 2001, AJ, 121, 2950

Larsen, S. S., Brodie, J. P., Huchra, J. P., et al. 2001, AJ, 121, 2974

Lauer, T. R., Ajhar, E. A., Byun, Y., et al. 1995, AJ, 110, 2622

Loewenstein, M., \& Mushotzky, F. 2002 [astro-ph/0208090]

McLaughlin, D. E., Harris, W. E., \& Hanes, D. A. 1993, ApJ, 409, L45

Nakazawa, K., Makashima, K., Fukazawa, Y., \& Tamura, T. 2000, PASJ, 52, 623

Pedersen, K., Yoshii, Y., \& Sommer-Larsen, J. 1997, ApJ, 485, L17

Poulain, P., \& Nieto, J.-L. 1994, A\&AS, 103, 573

Pritchet, C. J., \& Harris, W. E. 1990, ApJ, 355, 410

Prugniel, P., \& Simien, F. 1996, A\&A, 309, 749

Reid, N., Boisson, C., \& Sansom, E. A. 1994, MNRAS, 269, 713

Rhode, K. L., \& Zepf, S. E. 2001, AJ, 121, 210

Richtler, T., Dirsch, B., Gebhardt, K., et al. 2003, AJ, submitted

Rieke, G. H., \& Lebofsky, M. J. 1985, ApJ, 288, 618

Sandage, A. 1975, ApJ, 202, 563

Sandage, A., \& Betge, J. 1994, The Carnegie atlas of galaxies, vol. 1, Carnegie Institution of Washington

Schlegel, D., Finkbeiner, D., \& Davis, M. 1998, ApJ, 500, 525

Secker, J., Geisler, D., Mc Laughlin, D. E., \& Harris, W. E. 1995, AJ, 109,1019

Tonry, J. L., Dressler, A. D., \& Blakeslee, J. P. 2001, AJ, 546, 681

Tully, R. B. 1982, ApJ, 257, 389

White, S. D. M. 1987, in Structure and Dynamics of Elliptical Galaxies, ed. T. de Zeeuw (Dordrecht: D. Reidel), 263

Zepf, S. E., Ashman, K. M., \& Geisler, D. 1995, ApJ, 443, 570 